BIOCYT Biología, Ciencia y Tecnología, 13: 934-940, 2020.

ISSN: 2007-2082

DOI: $10.22201 /$ fesi.20072082.\%Y.\%v.75519

http://revistas.unam.mx/index.php/biocyt

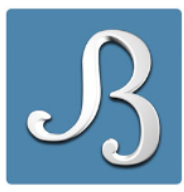

Publicada en la Facultad de Estudios Superiores Iztacala, Universidad Nacional Autónoma de México

\title{
ESTIMACIÓN PUNTUAL DE LA RELACIÓN FECUNDIDAD- LONGITUD DE GRAPTOCORIXA ABDOMINALIS (HEMIPTERA, CORIXIDAE) EN EL ESTANQUE PISCÍCOLA JORGE FACIO, ESTADO DE MÉXICO, MÉXICO
}

\section{PUNTUAL ESTIMATION OF THE RELATIONSHIP LENGTH- FECUNDITY OF GRAPTOCORIXA ABDOMINALIS (HEMIPTERA, CORIXIDAE) IN THE PISCICOLE POND JORGE FACIO, ESTADO DE MEXICO, MEXICO}

\footnotetext{
Gilberto Contreras Rivero $₫ 1$, Viridiana Arista Tapia루, Norma Angélica Navarrete Salgado ${ }^{3}$, Nadia Martínez Velázquez ${ }^{4}$, Julia Abarca Ramírez ${ }^{5}$, Ismael Castillo Reyes ${ }^{6}$, Raúl Gutiérrez Hernández ${ }^{7}$, Miguel Olvera Márquez ${ }^{8}$ y Edgar Valdés Rojas ${ }^{9}$

Universidad Nacional Autónoma de México, Facultad de Estudios Superiores Iztacala. Av. De los Barrios, No. 1, Los Reyes Iztacala, Tlalnepantla, Estado de México, México. C. P. 54090.

1】gilcori0822@gmail.com,2viriarista2@gmail.com,3normaa@unam.mx,4nadiamarvel02@gmail.com, 5abarcaramirezjulia@gmail.com, 6isma.reyes89@gmail.com,7rgutierrezhernandez37@gmail.com , 8m.olveradb@gmail.com, ${ }^{9}$ edgar.val@comunidad.unam.mx
}

\begin{abstract}
Is analyzed in a timely manner the relationship lenght-fecundity of the species Graptocorixa abdominalis in the Jorge Facio pond, Estado de Mexico. Three sampling stations were located in the littoral area of the pond ( $\mathrm{A}, \mathrm{B}$ and $\mathrm{C})$ and each was measured: temperature of the water, $\mathrm{pH}$, conductivity, dissolved oxygen, alkalinity, hardness, and turbidity. The insects were captured with a network of $50 \times 30 \mathrm{~cm}$ frame spoon and sweeping an area of one square meter. 237 females were analyzed in total: 65 A station, 60 in the B station and station C 112. The largest number of eggs recorded in a female of $6.94 \mathrm{~mm}$ with 64 and the smaller number of eggs in a female of $6.60 \mathrm{~mm}$ with one egg. The higher ratio lenght-fecundity arose in the season $\mathrm{C}\left(\mathrm{F}=21.803 \mathrm{~L}^{-0.032}\right)$, followed by the $B$ station $\left(\mathrm{F}=16.284 \mathrm{~L}^{0.1097}\right)$ and A station $\left(\mathrm{F}=8.2802 \mathrm{~L}^{0.2725}\right)$. The average length of $\mathrm{G}$. abdominalis was $7.65 \mathrm{~mm}$ and fecundity averaged 24.3 eggs. It is concluded that the fecundity of the species $G$. abdominalis is determined significantly by the influence of the environmental parameters present in the Jorge Facio pond.
\end{abstract}

Key words: Ahuautle, Corixids, Hemiptera, Number of eggs, Piscicole ponds.

Manuscrito recibido el 12 de agosto de 2019, aceptado el 12 de febrero de 2020. 


\section{RESUMEN}

Se analiza de manera puntual la relación fecundidad-longitud de Graptocorixa abdominalis en el estanque piscícola Jorge Facio, Estado de México. Se ubicaron tres estaciones de muestreo en la zona litoral del estanque (A, B y C) y en cada una se midió: temperatura del agua, $\mathrm{pH}$, conductividad, oxígeno disuelto, alcalinidad, dureza y turbiedad. Los insectos fueron capturados con una red de cuchara de $50 \times 30 \mathrm{~cm}$ de marco y barriendo un área de un metro cuadrado. Se analizaron en total 237 hembras: 65 de la estación A, 60 en la estación B y 112 de la estación C. El mayor número de huevecillos se registró en una hembra de $6.94 \mathrm{~mm}$ con 64 y el menor número de huevecillos en una hembra de $6.60 \mathrm{~mm}$ con un huevecillo. La mayor relación fecundidad-longitud se presentó en la estación $C\left(F=21.803 \mathrm{~L}^{-0.032}\right)$, seguida de la estación $B\left(F=16.284 \mathrm{~L}^{0.1097}\right)$ y la estación A $\left(F=8.2802 \mathrm{~L}^{0.2725}\right)$. La longitud promedio de G. abdominalis fue $7.65 \mathrm{~mm}$ y la fecundidad promedio fue 24.3 huevecillos. Se concluye que los parámetros ambientales registrados en éste periodo de trabajo en cada estación de muestreo no presentaron fluctuaciones notables pero su influencia sobre la fecundidad de G. abdominalis fue considerable.

Palabras clave: Ahuautle, Coríxidos, estanque piscícola, Hemiptera, número de huevos.

\section{INTRODUCCIÓN}

Las comunidades rurales del Estado de México, poseen una escasa disponibilidad de agua para diversos usos, por lo que se utilizan diferentes sistemas de almacenamiento (bordos, jagüeyes y estanques entre otros). Estos sistemas son utilizados también para cultivar organismos acuáticos, siendo los peces los más utilizados. Lo anterior incrementa la utilidad de dichos sistemas ya que se obtiene proteína animal a bajo costo (Navarrete et al., 2004).

Asimismo, se pueden presentar también organismos tales como los hemípteros acuáticos; los cuales, son eslabones importantes en las tramas tróficas, ya que convierten la materia animal y vegetal en detritus, que es reincorporado a las tramas tróficas y es usado por otros organismos (Richard y Davis, 1984).

Dentro de los hemípteros acuáticos destacan por su abundancia en estos lugares los pertenecientes a la familia Corixidae; la cual, posee importancia desde el punto de vista alimentario, no solo para peces, sino también para aves, reptiles, quirópteros y como alimento humano (McCAfferty, 1981; Ramos-Elorduy, 1992; Adams, 1993).

No obstante lo anterior, los trabajos realizados con estos organismos en México son escasos (Peters, 1960; Peters y Spurgeon, 1971; Contreras et al., 2002, 2005, 2008). Debido a lo anterior el objetivo del presente trabajo fue estimar de forma puntual la relación fecundidad-longitud en $G$. abdominalis presente en el estanque piscícola Jorge Facio (JF) en Soyaniquilpan de Juárez, Estado de México. 


\section{MATERIALES Y MÉTODOS}

\section{Área de estudio}

El estanque Jorge Facio (JF) se localiza en el municipio de Soyaniquilpan de Juárez, Estado de México, entre los $20^{\circ} 07^{\prime} 14^{\prime \prime}$ a $20^{\circ} 07^{\prime} 4.9^{\prime \prime}$ latitud norte y $99^{\circ} 53^{\prime} 53^{\prime \prime}$ a $99^{\circ} 53^{\prime} 14.3^{\prime \prime}$ longitud oeste a una altitud de 2400 metros sobre el nivel del mar (msnm). Limita con el municipio de Jilotepec y el estado de Hidalgo. El clima es templado subhúmedo con verano fresco y lluvioso y con poca oscilación térmica. La vegetación está formada principalmente de pastizal en las zonas planas y se pueden ver también organismos de Quercus, Cupressus y Eucalyptus. El estanque es alimentado por un canal de río y un arroyo de caudal permanente (INEGI, 2010).

Se seleccionaron de manera preferencial (Margalef, 1983) tres estaciones de muestreo en la zona litoral del estanque (A, B y C) el día 09 de marzo de 2013 y en cada una de ellas, se midió (in situ) la temperatura del agua con un termómetro digital Elite, el $\mathrm{pH}$ se midió con un potenciómetro digital Cole Parmer, la conductividad con un conductivímetro de campo modelo Sprite 6000, el oxígeno con un oxímetro Cole Parmer, la dureza y la alcalinidad fueron determinadas mediante métodos colorimétricos de acuerdo a Clesceri et al. (1998). La turbidez del agua, se estimó con un turbidímetro de campo Lamotte 2020.

El material biológico fue capturado utilizando una red de cuchara de 50X30 $\mathrm{cm}$ de marco y barriendo un área de un metro cuadrado. Los organismos fueron colocados en bolsas de polietileno etiquetadas conteniendo formalina al 4\% (Gaviño et al., 1987). En el laboratorio fueron lavados con agua corriente para eliminar el exceso de formalina y se identificaron a nivel específico con ayuda de las claves de Hungerford (1948). Las hembras fueron medidas con ayuda de un vernier digital Stainless Hardened con precisión de centésimas de mm y disectadas con ayuda de agujas de disección curvas. Para realizar el conteo de huevecillos de cada hembra, estas se colocaron bajo un microscopio estereoscópico marca Velab modelo VE-S3. La estimación de la relación fecundidadlongitud se realizó mediante una regresión potencial siguiendo el criterio de Bagenal y Tesch (1978) $\mathrm{F}=\mathrm{aL}$

Donde $\mathrm{F}$ es la fecundidad, a es el factor de condición, que en este caso corresponde al número de huevecillos, L es la longitud y $\mathrm{b}$ representa el tipo de crecimiento.

\section{RESULTADOS}

Los parámetros físicos y químicos registrados en el estanque JF, se muestran en la Tabla 1.

Tabla 1. Parámetros físicos y químicos en el estanque JF. Table 1. Physical and chemical parameters in the JF pond.

\begin{tabular}{lccc} 
Parámetros & Estación A & Estación B & Estación \\
& & & $\mathrm{C}$ \\
\hline Temperatura del agua $\left({ }^{\circ} \mathrm{C}\right)$ & 18.5 & 18.6 & 16.7 \\
$\mathrm{pH}$ & 8.0 & 8.0 & 7.9 \\
Conductividad $(\mu \mathrm{S} / \mathrm{cm})$ & 343.0 & 341.0 & 340.0 \\
Oxígeno $(\mathrm{mg} / \mathrm{L})$ & 7.12 & 8.9 & 7.3 \\
Alcalinidad $(\mathrm{mg} / \mathrm{L})$ & 110.0 & 113.0 & 108.0 \\
Dureza $(\mathrm{mg} / \mathrm{L})$ & 150.0 & 148.0 & 147.0 \\
Turbiedad $(\mathrm{UNF})$ & 75.5 & 48.2 & 60.3
\end{tabular}

Se revisaron en total 270 hembras de la especie Graptocorixa abdominalis (Say, 1832), 33 de éstas no presentaron ningún huevecillo por lo que no fueron consideradas en el análisis numérico. 
Los resultados señalados corresponden a un total de 237 hembras (65 en la estación A, 60 en la estación B y 112 en la estación C). La hembra más pequeña midió $4.62 \mathrm{~mm}$ y presentó seis huevecillos, mientras que la hembra más grande midió $7.93 \mathrm{~mm}$ y se observaron dos huevecillos. El menor número de huevecillos lo presentó una hembra de $6.6 \mathrm{~mm}$ con un huevecillo y el mayor número de huevecillos se presentó en una hembra de $6.94 \mathrm{~mm}$ con 64 huevecillos.

La longitud promedio registrada fue $7.65 \mathrm{~mm}$ y la fecundidad promedio fue 24.3 huevecillos. El número total de huevecillos contabilizados fue 5737. Los modelos de la relación fecundidad-longitud mostraron a la estación $\mathrm{C}$ con la mayor fecundidad $\left(\mathrm{F}=21.803 \mathrm{~L}^{-0.032}\right)$, seguida de la estación $B\left(F=16.284 L^{0.1097}\right)$ y la estación A $\left(F=8.2802 L^{0.2725}\right)$ (Figs. 1-3).

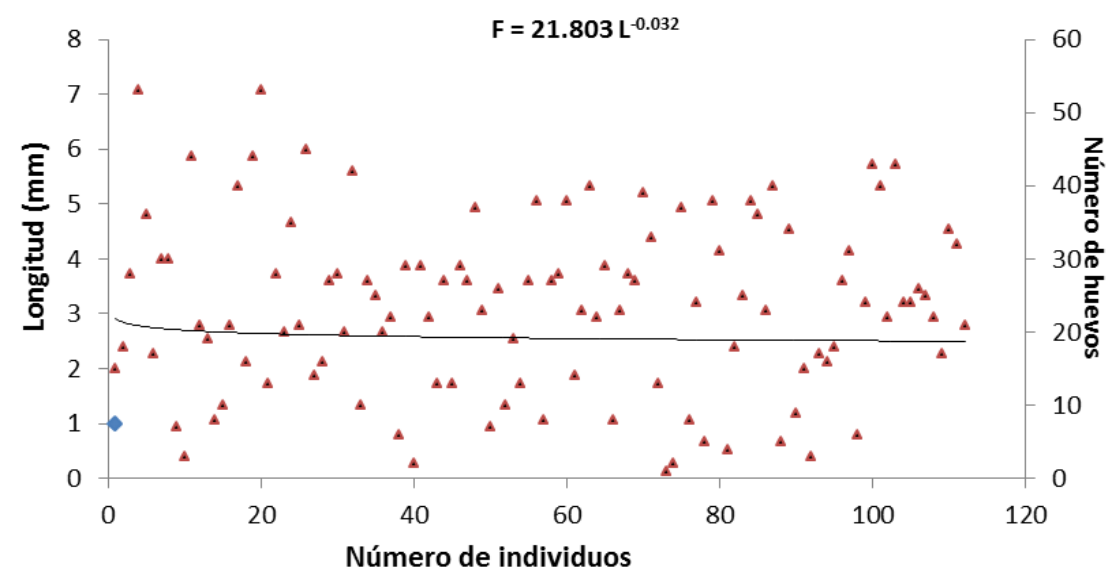

Fig. 1. Relación fecundidad-longitud en la estación C.

Fig. 1. Fecundity-length ratio at station $C$.

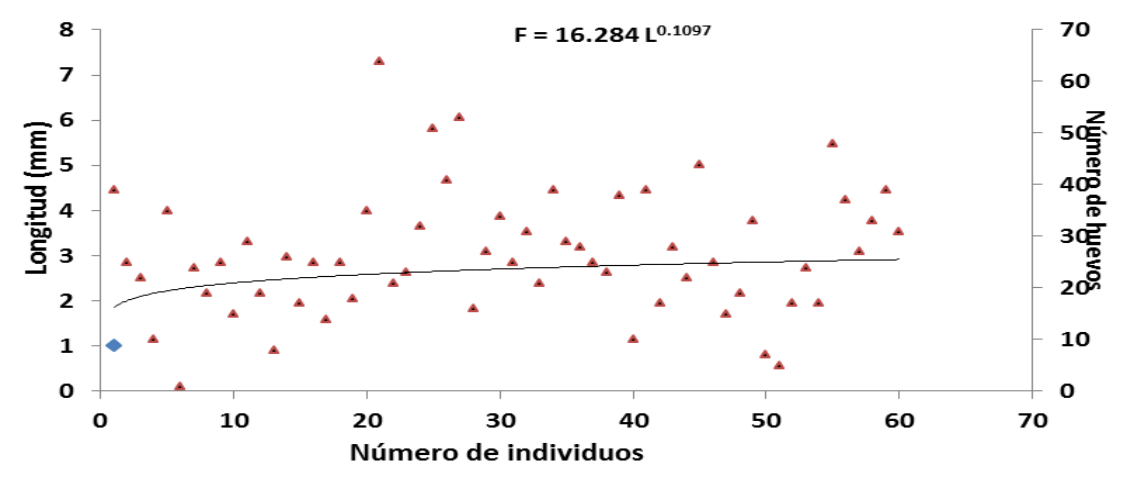

Fig. 2. Relación fecundidad-longitud en la estación B.

Fig. 2. Fecundity-length ratio at station B. 


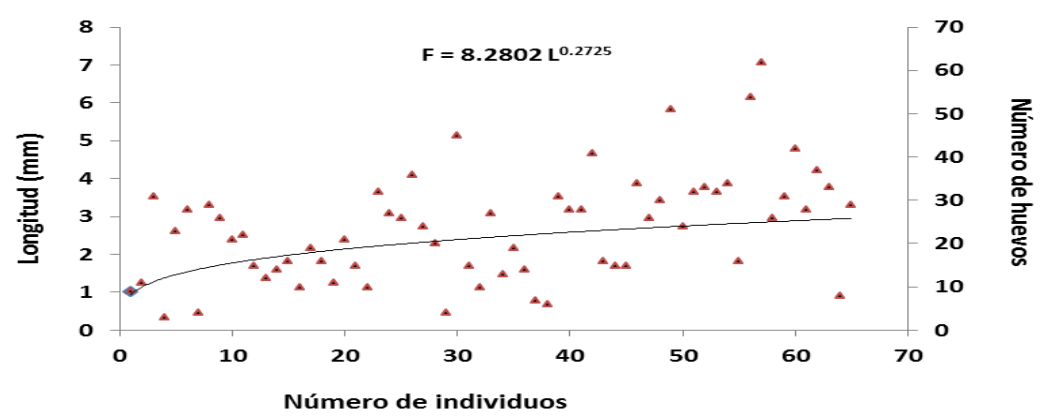

Fig. 3. Relación fecundidad-longitud en la estación A.

Fig. 3. Fecundity-length ratio at station A.

\section{DISCUSIÓN}

La mayor fecundidad se registró en la estación $C$, en donde se presentó la menor temperatura $\left(16.7^{\circ} \mathrm{C}\right)$, Hutchinson (1993) menciona que las especies con origen de tipo holártico, no se ven afectadas por temperaturas bajas respecto a aspectos reproductivos. En la estación B el valor de fecundidad fue menor en comparación con la estación C; esto posiblemente es debido a que la estación B presentó el mayor valor de temperatura $\left(18.6^{\circ} \mathrm{C}\right)$, lo que sí pudo afectar el proceso de fecundidad. Por otra parte, la concentración de oxígeno disuelto fue la más alta en ésta estación (8.9 $\mathrm{mg} / \mathrm{L}$ ) y la turbiedad fue la menor (48.2 UNF). Esta característica incrementa el proceso depredatorio en estos organismos, debido a que son fácilmente visibles para ciertos depredadores como peces, notonéctidos entre otros (Pajunen y Pajunen, 1992), incidiendo notablemente en su fecundidad. La estación A registró la menor fecundidad. En esta estación los valores de conductividad (343 micromhos/cm), dureza $(147 \mathrm{mg} / \mathrm{L})$, turbiedad (75.5 UNF) y oxígeno disuelto $(7.12 \mathrm{mg} / \mathrm{L})$, fueron los más bajos.

La conductividad y la dureza favorecen la disponibilidad de alimento para estos y otros organismos (Lind, 1985), Polhemus (1984) menciona que el nivel de turbiedad elevado impide el reconocimiento de los sexos en los coríxidos por lo que un macho puede aparearse accidentalmente con otro macho, por lo que disminuye la fecundidad. Una concentración baja de oxígeno disuelto, también afecta este proceso ya que los huevecillos requieren de una buena oxigenación para su desarrollo (Hutchinson, 1993).

El número de huevecillos presentes en G. abdominalis (1 a 64) es mayor al registrado por Pajunen y Pajunen (1991) para las especies Arctocorisa carinata (C. Sahlberg, 1819) y Callicorixa producta (Reuter, 1880) con intervalos de 9 a 12 y de 6 a 15, respectivamente, pero menor al observado por Peters y Spurgeon (1971) para la especie Krizousacorixa femorata (Guérin-Méneville, 1857) (40 a 972 huevecillos). Lo anterior depende de la cantidad de alimento disponible para las hembras (Kaitala, 1987) y también del tamaño del cuerpo de la hembra adulta que se ve drásticamente influido por la temperatura (Vannote y Sweeney, 1980).

Se puede concluir que pequeñas fluctuaciones en los parámetros ambientales registrados en el estanque influyen de forma considerable en la relación fecundidad-longitud de G. abdominalis. 


\section{AGRADECIMIENTOS}

Los autores agradecen la valiosa ayuda de los evaluadores.

\section{REFERENCIAS}

1. Adams R.A., 1993. Consumption of water boatmen (Hemiptera: Corixidae) by little brown bats, Myotis lucifugus. Bat Research News, 34(2-3): 66-67.

2. Bagenal T.B. y Tesch F.W., 1978. Age and growth. En: Bagenal T.B (Ed.), Methods for assessment of fish production in fresh waters, pp. 101-136. Oxford, UK, Blackwell Science Publications.

3. Clesceri L.S., Greenberg A.E., Trussell R.R. y Franson M.A.H. (Eds.) 1998. Métodos normalizados para el análisis de aguas potables y residuales. American Public Health Association, American Water Works Association, Water Pollution Control Federation (APHA, AWWA \& WPCF). Díaz de santos S. A. Madrid, España.

4. Contreras-Rivero G., Navarrete-Salgado NA., Elías-Fernández G. y Rojas-Bustamante M.L., 2002. Los coríxidos (Hemiptera, Heteroptera) del estanque piscícola denominado "JC" en Soyaniquilpan de Juárez, Estado de México. Revista Chapingo, Serie Ciencias Forestales y del Ambiente, 8: 113118.

5. Contreras-Rivero G., Camarillo de la Rosa G., Navarrete-Salgado N.A. y Elías-Fernández G., 2005. Corixidae (Hemiptera, Heteroptera) en el lago urbano del parque Tezozomoc, Azcapotzalco, México, D.F. Revista Chapingo, Serie Ciencias Forestales y del Ambiente, 11: 93-97.

6. Contreras-Rivero G., Navarrete-Salgado N.A. y Lara-Vázquez J.A., 2008. Hemípteros acuáticos en dos estanques piscícolas del Estado de México. Revista Chapingo, Serie Ciencias Forestales y del Ambiente, 14: 39-43.

7. Gaviño G.T., G., Juárez, C.L. y Figueroa H.H.T., 1987. Técnicas biológicas selectas de laboratorio y de campo. , México, LIMUSA.

8. Hungerford H.B., 1948. The Corixidae of the Western Hemisphere (Hemiptera). Kansas, University of Kansas Science Bulletin.

9. Hutchinson G.E., 1993. A treatise on limnology, Vol. 4., The zoobenthos. John. New York, USA, John Wiley \& Sons, Inc.

10. INEGI (Instituto Nacional de Geografía y Estadística), 2010. Marco Geoestadístico municipal 2010. Soyaniquilpan de Juárez. Versión 4.2.

11. Kaitala A. 1987. Dynamic life-history strategy of the waterstrider Gerris thoracicus as an adaptation to food and habitat variation. Oikos 48 (2): 125-131. DOI: 10.2307/3565848

12. Lind O.T., 1985. Handbook of common methods in limnology. Dubuque, Iowa, Kendall/Hunt Publishing company.

13. Margalef R., 1983. Limnología. Barcelona, Omega. 
14. McCafferty P.W., 1981. Aquatic entomology: The fishermen's and ecologists' illustrated guide to insects and their relatives. Boston, Massachusetts, Science Books International.

15. Navarrete S.N., Elías F.G., Contreras R.G., Rojas B.M. y Sánchez M.R., 2004. Piscicultura y ecología en estanques dulceacuícolas. México, AGT Editor S.A.

16. Pajunen V.I. y Pajunen I., 1991. Oviposition and egg cannibalism in rock-pool corixids (Hemiptera: Corixidae). Oikos, 60: 83-90. DOI: 10.2307/3544996

17. Pajunen V.I. y Pajunen I. 1992. Field evidence of intra- and interspecific predation in rock-pool corixids (Heteroptera, Corixidae). Entomologica Fennica, 3: 15-19. DOI: 10.33338/ef.83566

18. Peters W., 1960. Inheritance of asymmetry in a water-boatman (Krizousacorixa femorata). Nature, 186: 737. DOI: $10.1038 / 186737 \mathrm{a} 0$

19. Peters W. y Spurgeon J., 1971. Biology of the water-boatman Krizousacorixa femorata (Heteroptera: Corixidae). The American Midland Naturalist, 86 (1): 197-207. DOI: 10.2307/2423700

20. Polhemus T.J., 1984. Aquatic and semiaquatic Hemiptera. En: Merrit R.W. y Cummins W.K. (Eds.), An introduction to the aquatic insects of North America, pp. 231-255. Dubuque, Iowa,USA, Kendall/Hunt Publishing company.

21. Ramos-Elorduy J., 1992. Los insectos como fuente de proteínas en el futuro. México, LIMUSA.

22. Richards O.W. y Davies R.G., 1984. Tratado de entomología Imms, Vol. 2., Barcelona, Omega.

23. Vannote R.L. y Sweeney B.W., 1980. Geographic analysis of thermal equilibria: A conceptual model for evaluating the effect of natural and modified thermal regimen on aquatic insects communities. The American Naturalist, 115: 667-695.

\section{BIOCYT Biología, Ciencia y Tecnología, se encuentra actualmente indexada en}
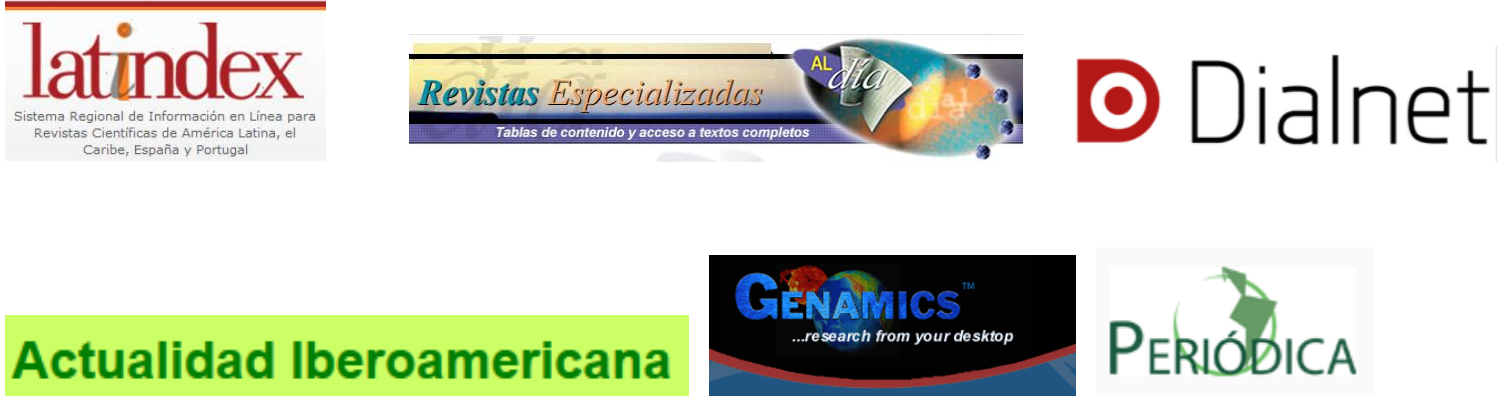

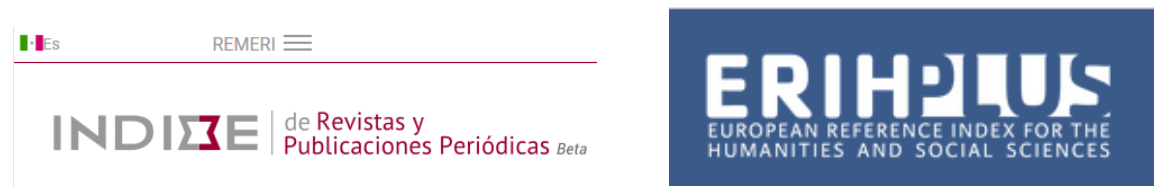

alojada en los repositorios
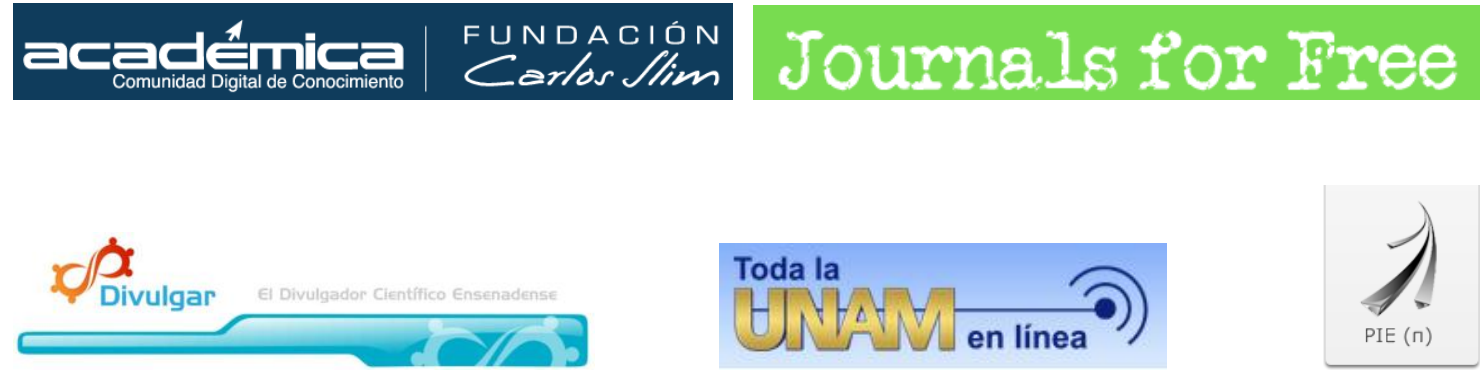

y en bases electrónicas de bibliotecas

DD $\begin{aligned} & \text { LEIBNIZ-INFORMATIONSZENTRUM } \\ & \text { TECHNIK UND NATURWISSENSCHAFTEN } \\ & \text { UNIVERSITÄTSBIBLIOTHEK }\end{aligned}$
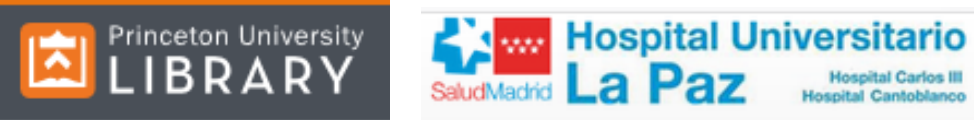

Universität Vechta

University of Vechto 
BIOCYT, 13: 934-940, 2020. Gilberto Contreras Rivero. Relación fecundidad-longitud de Graptocorixa abdominalis.

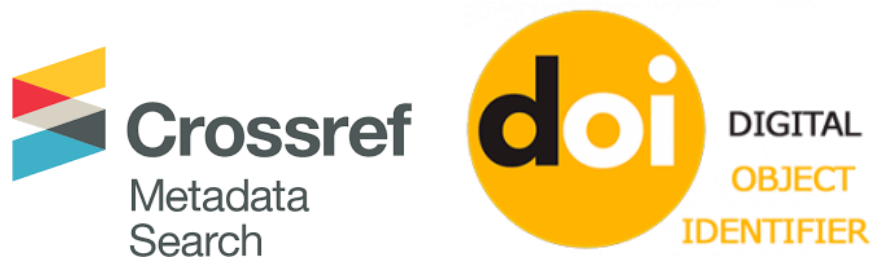

